\title{
Sediment Transport and Deposition in Fishercap Lake and the Swiftcurrent Valley, Glacier National Park, Montana, USA
}

Kelly MacGregor (Macalester College), Amy Myrbo (University of Minnesota), Diala Abboud (Macalester College), Elizaveta Atalig (Wesleyan University), Etienne Chenevert (Macalester College), Elizabeth Moore (Washington and Lee Univer



